\title{
A NOTE ON HAUSDORFF MEASURES OF SELF-SIMILAR SETS IN $\mathbf{R}^{d}$
}

\author{
Cai-Yun Ma and Yu-Feng Wu
}

The Chinese University of Hong Kong, Department of Mathematics

Shatin, Hong Kong; cyma@math.cuhk.edu.hk

South China University of Technology, Department of Mathematics

Guangzhou 510641, P. R. China; yufengwu@scut.edu.cn, and

The Chinese University of Hong Kong, Department of Mathematics, Shatin, Hong Kong

\begin{abstract}
We prove that for all $s \in(0, d)$ and $c \in(0,1)$ there exists a self-similar set $E \subset \mathbf{R}^{d}$ with Hausdorff dimension $s$ such that $\mathcal{H}^{s}(E)=c|E|^{s}$. This answers a question raised by Zhiying Wen [16].
\end{abstract}

\section{Introduction}

In this paper, we investigate Hausdorff measures of self-similar sets in $\mathbf{R}^{d}$. Recall that given a finite family of contracting similitudes $\Phi=\left\{\phi_{i}\right\}_{i=1}^{\ell}$ on $\mathbf{R}^{d}$, there is a unique non-empty compact set $K \subset \mathbf{R}^{d}$ satisfying $K=\bigcup_{i=1}^{\ell} \phi_{i}(K)$. We call $\Phi$ an iterated function system (IFS) of similitudes and $K$ the self-similar set generated by $\Phi$. Moreover, given a probability vector $\mathbf{p}=\left(p_{1}, \ldots, p_{\ell}\right)$, i.e. all $p_{i}>0$ and $\sum_{i=1}^{\ell} p_{i}=1$, there is a unique Borel probability measure $\nu$ supported on $K$ satisfying

$$
\nu=\sum_{i=1}^{\ell} p_{i} \nu \circ \phi_{i}^{-1} .
$$

We call $\nu$ the self-similar measure generated by $\Phi$ and $\mathbf{p}$. We refer the reader to $[9,7]$ for the examples and detailed properties of self-similar sets and self-similar measures.

We say that $\Phi$ satisfies the strong separation condition (SSC) if $\phi_{i}(K) \cap \phi_{j}(K)=\emptyset$ for all distinct $i, j \in\{1, \ldots, \ell\}$. Similarly, we say that $\Phi$ satisfies the open set condition (OSC) if there exists a non-empty bounded open set $V \subset \mathbf{R}^{d}$ such that $\phi_{1}(V), \ldots, \phi_{\ell}(V)$ are disjoint subsets of $V$. Under the OSC, it is well-known that the Hausdorff dimension of $K$, denoted by $\operatorname{dim}_{\mathrm{H}} K$, equals the similarity dimension of $\Phi$, i.e. the positive number $s$ satisfying $\sum_{i=1}^{\ell} r_{i}^{s}=1$, where $r_{i} \in(0,1)$ is the contraction ratio of $\phi_{i}, i=1, \ldots, \ell$. Also, the $s$-dimensional Hausdorff measure of $K$ satisfies that $0<\mathcal{H}^{s}(K) \leq|K|^{s}$, here and afterwards for $A \subset \mathbf{R}^{d},|A|$ stands for the diameter of $A$. Moreover, it is known that

$$
\left.\mathcal{H}^{s}\right|_{K}=\mathcal{H}^{s}(K) \mu
$$

where $\left.\mathcal{H}^{s}\right|_{K}$ stands for the restriction of the $s$-dimensional Hausdorff measure on $K$, $\mu$ is the self-similar measure generated by $\Phi$ and the probability vector $\left(r_{1}^{s}, \ldots, r_{\ell}^{s}\right)$. We call $\mu$ the natural self-similar measure on $K$. See [9] for the proofs of the above facts.

Nevertheless, given a self-similar set $K \subset \mathbf{R}^{d}$ satisfying the OSC with $\operatorname{dim}_{\mathrm{H}} K=$ $s$, it is often challenging to determine the exact value of $\mathcal{H}^{s}(K)$. When $d=1$, this problem was first studied independently by Marion $[11,12]$ and Ayer and Strichartz

https://doi.org/10.5186/aasfm.2021.4661

2020 Mathematics Subject Classification: Primary 28A78, 28A80.

Key words: Self-similar sets, Hausdorff measure. 
[1]. They computed the exact value of $\mathcal{H}^{s}(K)$ under certain additional hypothesis. Their method is based on the relation (1.1) and the convex density theorem for Hausdorff measures (see [6, Theorem 2.3]). When $d>1$ and $s>1$ is not an integer, despite numerous studies, not a single example is known for which the exact value of $\mathcal{H}^{s}(K)$ is computed; see $[2,3,4,17]$ for related works. In this case, it is known that $\mathcal{H}^{s}(K)<|K|^{s}$ if the convex hull of $K$ is a polytope (see [8, Corollary 1.6]). It remains an open question whether $\mathcal{H}^{s}(K)<|K|^{s}$ for any self-similar set $K \subset \mathbf{R}^{d}$ with similarity dimension $s>1$. Regarding of this problem, Zhiying Wen raised the following question in [16].

Question 1.1. Let $s>1$ and $\epsilon \in(0,1)$. Does there exist a self-similar set $K$ with similarity dimension $s$ such that $\mathcal{H}^{s}(K)>(1-\epsilon)|K|^{s} ?^{1}$

In this paper, we give an affirmative answer to the above question by proving the following.

Theorem 1.2. For every $s \in(0, d)$ and $c \in(0,1)$, there exists a self-similar set $K \subset \mathbf{R}^{d}$ so that its generating IFS satisfies the SSC, $\operatorname{dim}_{\mathrm{H}} K=s$ and $\mathcal{H}^{s}(K)=c|K|^{s}$.

Our strategy of the proof is as follows: given $s \in(0, d)$ and $\epsilon \in(0,1)$, we construct a family of IFSs $\left\{\Phi_{t}\right\}_{t \in[0,1]}$ with the corresponding self-similar sets $\left\{K_{t}\right\}_{t \in[0,1]}$ such that: (1) for each $t \in[0,1], \Phi_{t}$ satisfies the $\mathrm{SSC}, \operatorname{dim}_{\mathrm{H}} K_{t}=s$ and $\left|K_{t}\right|=1$; (2) the mapping $t \mapsto \mathcal{H}^{s}\left(K_{t}\right)$ is continuous; (3) $\mathcal{H}^{s}\left(K_{0}\right)>1-\epsilon$; (4) $\mathcal{H}^{s}\left(K_{1}\right)<\epsilon$. See Proposition 2.2 for the details. A key part is the proof of (3), in which we apply the isodiametric inequality (see Lemma 2.4). Then Theorem 1.2 follows from the above result and a result in [13] about the continuity of Hausdorff measures of self-similar sets satisfying the SSC with respect to the defining data of the IFSs.

\section{Proof of Theorem 1.2}

Our proof of Theorem 1.2 is based on the following result.

Lemma 2.1. [14] Let $K \subset \mathbf{R}^{d}$ be a self-similar set generated by an IFS $\Phi=$ $\left\{\phi_{i}\right\}_{i=1}^{\ell}$ which satisfies the $S S C$ with $\operatorname{dim}_{\mathrm{H}} K=s$. Let $\mu$ be the natural self-similar measure on $K$. Set $\Delta=\min _{i \neq j} \operatorname{dist}\left(\phi_{i}(K), \phi_{j}(K)\right)$. Then we have

$$
\begin{aligned}
\mathcal{H}^{s}(K)^{-1} & =\max \left\{\frac{\mu(U)}{||^{s}}: U \subset \mathbf{R}^{d} \text { is compact and convex }\right\} \\
& =\max \left\{\frac{\mu(U)}{||^{s}}: U \subset \mathbf{R}^{d} \text { is compact and convex with }|U| \geq \Delta\right\} .
\end{aligned}
$$

In particular, the above maximums are both attained.

We remark that when $d=1$, Lemma 2.1 was proved earlier in $[11,15,1]$, where it was used to compute Hausdorff measures of self-similar sets in $\mathbf{R}$ under certain additional hypothesis. Moreover, Lemma 2.1 is not explicitly stated in [14], but it can be easily deduced from the results of [14]. Indeed, it was proved in [14,

\footnotetext{
${ }^{1}$ It is known that for each $s \in(0,1]$, there exists a self-similar set $K \subset \mathbb{R}$ with similarity dimension $s$ such that $\mathcal{H}^{s}(K)=|K|^{s}$ (see e.g. $[11,1]$ ).
} 
Corollaries 1.5-1.6] that

$$
\begin{aligned}
\mathcal{H}^{s}(K)^{-1} & =\sup \left\{\frac{\mu(U)}{|U|^{s}}: U \subset \mathbf{R}^{d} \text { is open and convex }\right\} \\
& =\sup \left\{\frac{\mu(U)}{|U|^{s}}: U \subset \mathbf{R}^{d} \text { is open and convex with }|U| \geq \Delta\right\} .
\end{aligned}
$$

Then Lemma 2.1 follows from the above equalities together with a standard compactness argument.

Based on Lemma 2.1, we establish the following result, which is an essential part in our proof of Theorem 1.2.

Proposition 2.2. For every $s \in(0, d)$ and $\epsilon \in(0,1)$, there exist $r \in(0,1)$, $\ell \in \mathbf{N}$ and a family of IFSs $\Phi_{t}=\left\{\phi_{t, i}(x)=r x+a_{i}(t)\right\}_{i=1}^{\ell}(t \in[0,1])$ on $\mathbf{R}^{d}$ with $a_{1}, \ldots, a_{\ell}:[0,1] \rightarrow \mathbf{R}^{d}$ being continuous functions such that the following statements hold:

(i) For each $t \in[0,1], \Phi_{t}$ satisfies the $S S C$ and its attractor, denoted as $K_{t}$, has dimension $s$ and diameter 1 .

(ii) $\mathcal{H}^{s}\left(K_{0}\right)>1-\epsilon$ and $\mathcal{H}^{s}\left(K_{1}\right)<\epsilon$.

To prove Proposition 2.2, we first give an elementary lemma.

Lemma 2.3. Let $0<s<d$ and $\epsilon>0$. Then there exists $N>0$ such that for all $n \geq N$ and all $x \in\left[\frac{1}{4 n}, 1\right]$,

$$
\frac{\left(x+\frac{\sqrt{d}}{n}\right)^{d}}{\left(1-\frac{\sqrt{d}}{2 n}\right)^{d} x^{s}}<1+\epsilon .
$$

Proof. Let $y_{0}>0$ be large enough such that for all $y \geq y_{0}$,

$$
\frac{\left(1+\frac{\sqrt{d}}{y}\right)^{d}}{\left(1-\frac{\sqrt{d}}{y}\right)^{d}}<1+\epsilon
$$

Then take $N>0$ large enough such that for all $n \geq N$,

$$
\frac{(1+4 \sqrt{d})^{d}}{\left(1-\frac{\sqrt{d}}{2 n}\right)^{d}}\left(\frac{y_{0}}{n}\right)^{d-s}<1+\epsilon
$$

Notice that such $N$ exists since $s<d$. Let $n \geq N$ and $x \in\left[\frac{1}{4 n}, 1\right]$. If $n x \geq y_{0}$, then $2 n>n x \geq y_{0}$ and so by $(2.3)$,

$$
\frac{\left(x+\frac{\sqrt{d}}{n}\right)^{d}}{\left(1-\frac{\sqrt{d}}{2 n}\right)^{d} x^{s}}=\frac{\left(1+\frac{\sqrt{d}}{n x}\right)^{d}}{\left(1-\frac{\sqrt{d}}{2 n}\right)^{d}} x^{d-s} \leq \frac{\left(1+\frac{\sqrt{d}}{n x}\right)^{d}}{\left(1-\frac{\sqrt{d}}{n x}\right)^{d}} x^{d-s}<1+\epsilon .
$$


If $n x<y_{0}$, then

$$
\begin{array}{rlr}
\frac{\left(x+\frac{\sqrt{d}}{n}\right)^{d}}{\left(1-\frac{\sqrt{d}}{2 n}\right)^{d} x^{s}} & \left.\leq \frac{(x+4 \sqrt{d} x)^{d}}{\left(1-\frac{\sqrt{d}}{2 n}\right)^{d} x^{s}} \quad \text { (since } \frac{1}{n} \leq 4 x\right) \\
& =\frac{(1+4 \sqrt{d})^{d}}{\left(1-\frac{\sqrt{d}}{2 n}\right)^{d}} x^{d-s} \\
& \leq \frac{(1+4 \sqrt{d})^{d}}{\left(1-\frac{\sqrt{d}}{2 n}\right)^{d}}\left(\frac{y_{0}}{n}\right)^{d-s} \quad\left(\text { since } n x<y_{0}\right) \\
& <1+\epsilon \quad \quad \text { (by }(2.4)) .
\end{array}
$$

This completes the proof of the lemma.

Let $\mathcal{L}^{d}$ denote the $d$-dimensional Lebesgue measure on $\mathbf{R}^{d}$. The following standard isodiametric inequality plays a key role in our proof of Proposition 2.2.

Lemma 2.4. [5, Theorem 2.4] For every Lebesgue measurable set $A \subset \mathbf{R}^{d}$,

$$
\mathcal{L}^{d}(A) \leq \omega_{d} 2^{-d}|A|^{d},
$$

where $\omega_{d}$ denotes the Lebesgue measure of a unit ball in $\mathbf{R}^{d}$.

For $x=\left(x_{1}, \ldots, x_{d}\right) \in \mathbf{R}^{d}$ and $\delta>0$, let $B(x, \delta)$ be the closed ball in $\mathbf{R}^{d}$ centered at $x$ of radius $\delta$, and let $Q(x, \delta)$ denote the cube $\prod_{i=1}^{d}\left[x_{i}-\delta, x_{i}+\delta\right]$. For $A \subset \mathbf{R}^{d}$, let $\# A$ be the cardinality of $A$.

Proof of Proposition 2.2. Fix $s \in(0, d)$ and $\epsilon \in(0,1)$. We are going to construct the self-similar sets $K_{t}(t \in[0,1])$ in the ball $B:=B(0,1 / 2)$. For this purpose, let $N$ be as in Lemma 2.3. Pick a positive integer $n \geq N$ so that

$$
\frac{\omega_{d}\left(n-\frac{\sqrt{d}}{2}\right)^{d}-2}{(8 n+4)^{s}}>\epsilon^{-1} \text {. }
$$

Set $F=B \cap\left(\mathbf{Z}^{d} /(2 n)\right)$. Let $\ell=\# F$ and $b_{1}, \ldots, b_{\ell}$ be the different elements of $F$ with

$$
b_{1}=(-1 / 2,0, \ldots, 0), \quad b_{2}=(1 / 2,0, \ldots, 0) .
$$

Then by a simple volume argument (see e.g. [10, p. 17]),

$$
\ell \geq \omega_{d}\left(n-\frac{\sqrt{d}}{2}\right)^{d}
$$

Let $r=\ell^{-1 / s}$. Then by $(2.5)-(2.6)$,

$$
(8 n+4) r=(8 n+4) \ell^{-1 / s} \leq(8 n+4) \omega_{d}^{-1 / s}\left(n-\frac{\sqrt{d}}{2}\right)^{-d / s}<\epsilon^{1 / s}<1 .
$$

In particular, $8 n r<1$.

For $t \in[0,1]$, let $\Phi_{t}=\left\{\phi_{t, i}(x)=r x+a_{i}(t)\right\}_{i=1}^{\ell}$, where $a_{1}(t)=(1-r) b_{1}, a_{2}(t)=(1-r) b_{2}, a_{i}(t)=(1-r)(8 n r)^{t} b_{i} \quad$ for $i \in\{3, \ldots, \ell\}$. 
Let $K_{t}$ be the attractor of $\Phi_{t}$. Notice that $a_{1}, \ldots, a_{\ell}:[0,1] \rightarrow \mathbf{R}^{d}$ are continuous functions. Clearly, for each $1 \leq i \leq \ell$, the fixed point of $\phi_{t, i}$ is $a_{i}(t) /(1-r)$. In particular, $b_{1}, b_{2}$ are the fixed points of $\phi_{t, 1}$ and $\phi_{t, 2}$, respectively. Hence $b_{1}, b_{2} \in K_{t}$. Since $8 n r<1$, it is not difficult to check that for each $t \in[0,1], \phi_{t, i}(B)(i=1, \ldots, \ell)$ are pairwise disjoint and contained in $B$. This implies that $K_{t} \subset B$ (see e.g. [7]) and so $\left|K_{t}\right| \leq|B|=1$, and $\Phi_{t}$ satisfies the $\mathrm{SSC}$ with $\operatorname{dim}_{\mathrm{H}} K_{t}=s$. Since $b_{1}, b_{2} \in K_{t}$, $\left|K_{t}\right| \geq\left\|b_{1}-b_{2}\right\|=1$. Hence $\left|K_{t}\right|=1$. This proves the part (i) of the proposition. In the following we prove that $\mathcal{H}^{s}\left(K_{0}\right)>1-\epsilon$ and $\mathcal{H}^{s}\left(K_{1}\right)<\epsilon$.

For $t \in\{0,1\}$, let $\mu_{t}$ be the natural self-similar measure on $K_{t}$. That is, $\mu_{t}$ is the unique Borel probability measure supported on $K_{t}$ satisfying

$$
\mu_{t}=\sum_{i=1}^{\ell} r^{s} \mu_{t} \circ\left(\phi_{t, i}\right)^{-1} .
$$

We first show that $\mathcal{H}^{s}\left(K_{0}\right)>1-\epsilon$. Recall that $\Phi_{0}=\left\{\phi_{0, i}(x)=r x+(1-r) b_{i}\right\}_{i=1}^{\ell}$. See Figure 1(a) for an illustration of the locations of $b_{1}, \ldots, b_{\ell}$, which are the fixed points of the elements of $\Phi_{0}$.

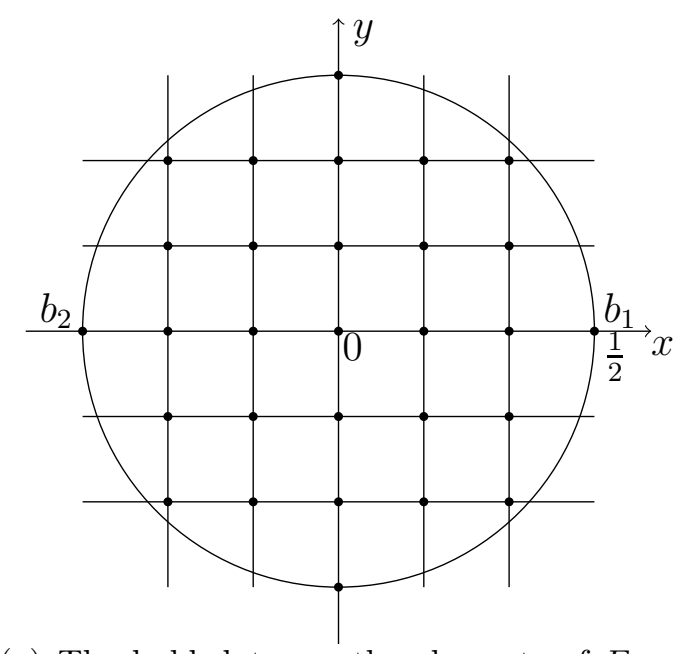

(a) The bold dots are the elements of $F$ and also the fixed points of the similitudes in $\Phi_{0}$.

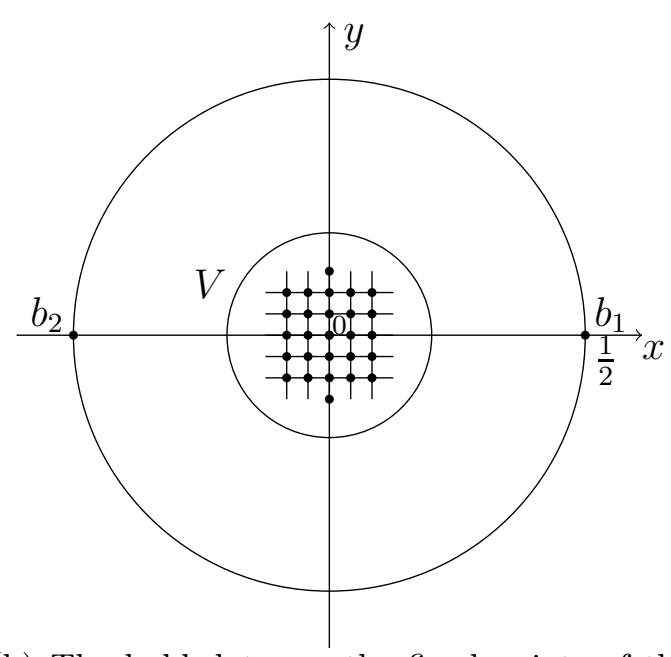

(b) The bold dots are the fixed points of the similitudes in $\Phi_{1}$.

Figure 1. $\Phi_{0}$ and $\Phi_{1}, n=3$.

Since $\left|K_{0}\right|=1$ and $8 n r<1$, we see that for $i \in\{1, \ldots, \ell\}, \phi_{0, i}\left(K_{0}\right)$ is contained in the interior of $Q\left(b_{i}, 1 /(4 n)\right)$. In particular, $Q\left(b_{i}, 1 /(4 n)\right) \cap \phi_{0, j}\left(K_{0}\right)=\emptyset$ for any $i, j \in\{1, \ldots, \ell\}$ with $i \neq j$. Hence by $(2.8)$,

$$
\mu_{0}\left(Q\left(b_{i}, 1 /(4 n)\right)\right)=r^{s}=\ell^{-1}, \quad i=1, \ldots, \ell .
$$

By (2.2) there exists a compact convex set $U \subset \mathbf{R}^{d}$ such that

$$
\frac{\mu_{0}(U)}{|U|^{s}}=\mathcal{H}^{s}\left(K_{0}\right)^{-1}
$$

with $|U| \geq \min _{i \neq j} \operatorname{dist}\left(\phi_{0, i}\left(K_{0}\right), \phi_{0, j}\left(K_{0}\right)\right)$. Notice that for $i \in\{1, \ldots, \ell\}, b_{i}$ is the fixed point of $\phi_{0, i}$ and so $b_{i} \in \phi_{0, i}\left(K_{0}\right)$. Hence for all $i \neq j$, since $\left|\phi_{0, i}\left(K_{0}\right)\right|=$ $\left|\phi_{0, j}\left(K_{0}\right)\right|=r$ and $8 n r<1$, we have by the triangle inequality,

$$
\operatorname{dist}\left(\phi_{0, i}\left(K_{0}\right), \phi_{0, j}\left(K_{0}\right)\right) \geq\left\|b_{i}-b_{j}\right\|-2 r \geq \frac{1}{2 n}-2 r>\frac{1}{4 n} \text {. }
$$


It follows that $|U| \geq \frac{1}{4 n}$. On the other hand, since $K_{0} \subset B$, we have $\mu_{0}(U)=$ $\mu_{0}(U \cap B)$ and so

$$
\frac{\mu_{0}(U)}{|U|^{s}} \leq \frac{\mu_{0}(U \cap B)}{|U \cap B|^{s}}
$$

However, since $U \cap B$ is compact and convex, by (2.1) and (2.10) we see that the equality holds in (2.11). Therefore $|U|=|U \cap B|$. Hence replacing $U$ by $U \cap B$ if necessary, we can assume that $U \subset B$ and thus $|U| \leq 1$. So we have

$$
\frac{1}{4 n} \leq|U| \leq 1
$$

Let $m=\# \mathcal{F}$, where $\mathcal{F}:=\{Q(b, 1 /(4 n)): b \in F, Q(b, 1 /(4 n)) \cap U \neq \emptyset\}$. Then by $(2.9)$ and $(2.6)$,

$$
\mu_{0}(U) \leq m \ell^{-1} \leq m \omega_{d}^{-1}\left(n-\frac{\sqrt{d}}{2}\right)^{-d} .
$$

On the other hand, notice that each cube in $\mathcal{F}$ is of diameter $\sqrt{d} /(2 n)$ and so it is contained in the closed $\sqrt{d} /(2 n)$-neighborhood of $U$, which we denote by $\overline{\mathbf{V}}_{\sqrt{d} /(2 n)}(U)$, and the intersection of any two different cubes in $\mathcal{F}$ has zero Lebesgue measure. Hence by a simple volume argument and the isodiametric inequality (see Lemma 2.4),

$$
m\left(\frac{1}{2 n}\right)^{d} \leq \mathcal{L}^{d}\left(\overline{\mathbf{V}}_{\sqrt{d} /(2 n)}(U)\right) \leq \omega_{d} 2^{-d}\left(|U|+\frac{\sqrt{d}}{n}\right)^{d} .
$$

Now by (2.13)-(2.14) and Lemma 2.3 (in which we take $|U|=x$ and recall (2.12)),

$$
\frac{\mu_{0}(U)}{|U|^{s}} \leq \frac{\left(|U|+\frac{\sqrt{d}}{n}\right)^{d}}{\left(1-\frac{\sqrt{d}}{2 n}\right)^{d}|U|^{s}}<1+\epsilon .
$$

This combining with $(2.10)$ yields that $\mathcal{H}^{s}\left(K_{0}\right)>1 /(1+\epsilon)>1-\epsilon$.

Finally, we show that $\mathcal{H}^{s}\left(K_{1}\right)<\epsilon$. Recall that $\Phi_{1}$ consists of the similitudes $\phi_{1,1}(x)=r x+(1-r) b_{1}, \phi_{1,2}(x)=r x+(1-r) b_{2}$ and $\phi_{1, k}(x)=r x+(1-r) 8 n r b_{k}$ for $k \in\{3, \ldots, \ell\}$. Let $V=B(0,(4 n+1) r)$. Since $(8 n+4) r<1$ (see $(2.7))$, it is easily checked that $\phi_{1,1}\left(K_{1}\right), \phi_{1,2}\left(K_{1}\right)$ are both disjoint from $V$, and $\phi_{1, k}\left(K_{1}\right) \subset V$ for $k \in\{3, \ldots, \ell\}$. See Figure $1(\mathrm{~b})$ for an illustration of $V$ and the locations of the fixed points of the elements of $\Phi_{1}$. Then by $(2.5)-(2.6)$,

$$
\frac{\mu_{1}(V)}{|V|^{s}}=\frac{1-2 r^{s}}{(8 n+2)^{s} r^{s}}=\frac{\ell-2}{(8 n+2)^{s}} \geq \frac{\omega_{d}\left(n-\frac{\sqrt{d}}{2}\right)^{d}-2}{(8 n+2)^{s}}>\epsilon^{-1} .
$$

Hence $\mathcal{H}^{s}\left(K_{1}\right)<\epsilon$ by Lemma 2.1. This completes the proof of the proposition.

With Proposition 2.2 in hand, we are ready to prove Theorem 1.2. The proof is a direct consequence of Proposition 2.2 combined with a continuity result in [13] about Hausdorff measures of self-similar sets generated by IFSs satisfying the SSC.

Proof of Theorem 1.2. Fix $s \in(0, d)$ and $c \in(0,1)$. Let $\epsilon>0$ be so small that $\epsilon<c<1-\epsilon$. Let $\Phi_{t}, K_{t}(t \in[0,1])$ be constructed as in Proposition 2.2. Since $a_{1}, \ldots, a_{\ell}:[0,1] \rightarrow \mathbf{R}^{d}$ are continuous functions, we easily see from [13, Theorem 1.2] that the mapping $t \mapsto \mathcal{H}^{s}\left(K_{t}\right)$ is continuous on $[0,1]$. Since $\mathcal{H}^{s}\left(K_{0}\right)>1-\epsilon, \mathcal{H}^{s}\left(K_{1}\right)<$ $\epsilon$ and $c \in(\epsilon, 1-\epsilon)$, the continuity of $t \mapsto \mathcal{H}^{s}\left(K_{t}\right)$ implies that $\mathcal{H}^{s}\left(K_{t_{0}}\right)=c$ for some 
$t_{0} \in[0,1]$. Therefore, $\mathcal{H}^{s}\left(K_{t_{0}}\right)=c\left|K_{t_{0}}\right|^{s}$ as $\left|K_{t_{0}}\right|=1$. Letting $K=K_{t_{0}}$ we complete the proof of Theorem 1.2.

Acknowledgements. The authors are grateful to their supervisor, De-Jun Feng, for many helpful discussions and suggestions. They also thank the two anonymous referees for their suggestions and comments to improve the paper. This research was partially supported by a HKRGC GRF grant (project 14301017) and the Direct Grant for Research in CUHK.

\section{References}

[1] Ayer, E., and R. S. Strichartz: Exact Hausdorff measure and intervals of maximum density for Cantor sets. - Trans. Amer. Math. Soc. 351:9, 1999, 3725-3741.

[2] DAI, X. R., W. H. He, and J. LuO: An isodiametric problem with additional constraints. - J. Math. Anal. Appl. 397:1, 2013, 1-8.

[3] DAI, X. R., W. H. He, J. LuO, and B. TAN: An isodiametric problem of fractal dimension. Geom. Dedicata 175:1, 2015, 79-91.

[4] Deng, J., H. RaO, and Z. Y. Wen: Hausdorff measure of Cartesian product of the ternary Cantor set. - Fractals 20:1, 2012, 77-88.

[5] Evans, L. C., and R. F. Gariepy: Measure theory and fine properties of functions. - CRC Press, Boca Raton, FL, 1992.

[6] Falconer, K. J.: The geometry of fractal sets. - Cambridge Univ. Press, Cambridge, 1985.

[7] Falconer, K. J.: Fractal geometry. Mathematical foundations and applications. Third edition. - John Wiley \& Sons, Ltd., Chichester, 2014.

[8] He, W. H., J. Luo, and Z. L. Zhou: Hausdorff measure and isodiametric inequalities. - Acta Math. Sinica (Chin. Ser.) 48:5, 2005, 939-946.

[9] Hutchinson, J. E.: Fractals and self-similarity. - Indiana Univ. Math. J. 30:5, 1981, 713-747.

[10] KrÄtzel, E.: Lattice points. - Kluwer Academic Publisher, Dordrecht, 1988.

[11] Marion, J. Mesure de Hausdorff d'un fractal à similitude interne. - Ann. Sc. Math. Québec $10: 1,1986,51-84$.

[12] Marion, J.: Mesures de Hausdorff d'ensembles fractals. - Ann. Sc. Math. Québec 11:1, 1987, $111-132$.

[13] Olsen, L.: Hausdorff and packing measure functions of self-similar sets: continuity and measurability. - Ergodic Theory Dynam. Systems 28:5, 2008, 1635-1655.

[14] Olsen, L.: Density theorems for Hausdorff and packing measures of self-similar sets. - Aequ. Math. 75:3, 2008, 208-225.

[15] Strichartz, R. S., A. Taylor, and T. Zhang: Densities of self-similar measures on the line. - Experiment. Math. 4:2, 1995, 101-128.

[16] WEN. Z. Y.: Private communication.

[17] Wu, M., and Z. L. Zhou: The Hausdorff measure of a Sierpiński carpet. - Sci. China Ser. A 42:7, 1999, 673-680.

Received 24 July 2020 • Accepted 25 January 2021 